\title{
相界上裂纹的两个奇异指数 *
}

考虑如图 1 所示的半无限长平面裂纹问 题. 裂纹与折线相界相交于相界拐点上, 裂 纹沿长线与相界的夹角分别为 $\lambda^{+} 、 \lambda^{-}$, 荷载 $P 、 Q$ 作用在远离裂纹尖端处,两相材料均为 均匀各向同性的弹性材料, 剪切模量和泊松 比分别为 $G_{1} 、 G_{2}$ 和 $\nu_{1} 、 \nu_{2}$.

以裂纹面和相界为边界将全平面分成三 个区域, 如图 1. 设每个区域在裂纹尖端附 近有复势解:

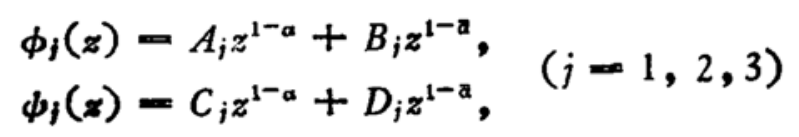

$A_{i} 、 B_{j} 、 C_{i} 、 D_{j}$ 为常数, $z=x+i y, \alpha$ 为应力 奇异指数, 则由

$$
\begin{gathered}
\left(\sigma_{\theta}\right)_{i}+\left(\sigma_{r}\right)_{i}-2\left[\phi_{i}^{\prime}(z)+\overline{\phi_{i}^{\prime}(z)}\right], \\
\left(\sigma_{\theta}\right)_{i}-\left(\sigma_{r}\right)_{i}+2 i\left(\sigma_{r \theta}\right)_{i} \\
-e^{2 i \theta}\left[\bar{z} \phi_{i}^{\prime \prime}(z)+\phi_{i}^{\prime}(z)\right], \\
2 G_{i}\left[\left(u_{z}\right)_{i}+i\left(u_{y}\right)_{i}\right] \\
-\kappa_{j} \phi_{i}(z)-z \overline{\phi_{i}^{\prime}(z)}-\overline{\phi_{j}(z)} .
\end{gathered}
$$

可得到应力和位移场, 其中 $\kappa_{i}-3-4 \nu_{i}$ (平 面应变). 应用裂纹面上应力为零和相界上 应力、位移连续的条件, 经过圥长的运算, 则 导出复势解中常数不全为零的条件:

$$
\begin{aligned}
& {\left[\frac{\overline{f^{+}}}{\Delta^{+}}-\frac{f^{-}}{\Delta^{-}}\right]\left[\frac{f^{+}}{\bar{\Delta}^{+}}-\frac{f^{-}}{\bar{\Delta}^{-}}\right]} \\
& \quad-\left[\frac{\overline{g^{+}}}{\Delta^{+}}-\frac{g^{-}}{\Delta^{-}}\right]\left[\frac{g^{+}}{\bar{\Delta}^{+}}-\frac{g^{-}}{\bar{\Delta}^{-}}\right]-0,
\end{aligned}
$$

其中

$$
\begin{aligned}
& f^{+}\left(\beta, \gamma, \lambda^{+}, \pi, \xi\right)-\xi\left(e^{2 i \lambda^{+}}-1\right) \\
& \quad \times[\gamma-1+\beta+2 \beta(\beta+1) \\
& \quad \times\left(\cos 2 \xi\left(\pi-\lambda^{+}\right)-1\right)-2 \beta^{2} \xi^{2} \\
& \left.\quad \times\left(\cos 2 \lambda^{+}-1\right)\right], \\
& \boldsymbol{g}^{+}\left(\beta, \gamma, \lambda^{+}, \pi, \xi\right)--1
\end{aligned}
$$

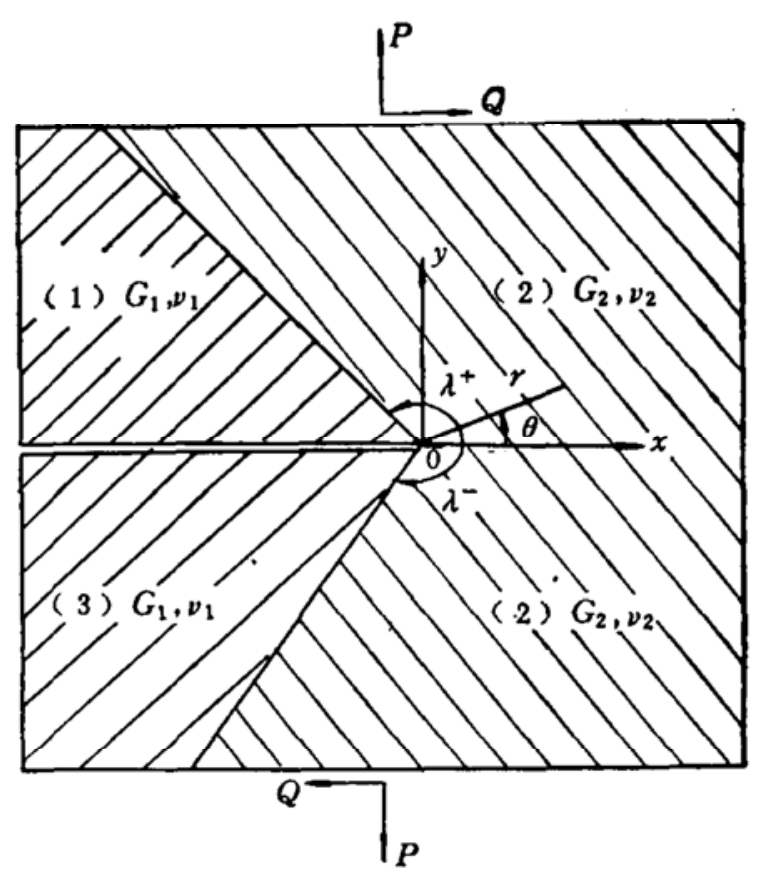

图 1 折线相界上的裂纹

$+2 \beta\left(\beta+\gamma e^{-2 i \lambda^{+} \xi}\right)\left[\cos 2 \xi\left(\pi-\lambda^{+}\right)\right.$

$\left.-1-\xi^{2}\left(\cos 2 \lambda^{+}-1\right)\right]+\gamma e^{-2 \pi i \xi}$

$+2 \beta\left(\cos 2 \xi\left(\pi-\lambda^{+}\right)-1\right)$

$-\beta e^{2 i \xi\left(x-2 \lambda^{+}\right)}+(1+\beta-\gamma) e^{-2 i \lambda^{+} \xi}$, $\Delta^{+}\left(\beta, \gamma, \lambda^{+}, \pi, \xi\right)$

$=1+2 \beta(\beta+1)(1-\cos 2 \xi$

$$
\left.\times\left(x-\lambda^{+}\right)\right)-2 \beta^{2} \xi^{2}\left(1-\cos 2 \lambda^{+}\right) \text {, }
$$

$f^{+}\left(\beta, \gamma, \lambda^{+}, x, \xi\right)-f^{+}\left(\beta, \gamma, \lambda^{+}, \pi, \xi\right)$, $\xi^{+}\left(\beta, \gamma, \lambda^{+}, \pi, \xi\right)-g^{+}\left(\beta, \gamma, \lambda^{+}, \pi, \xi\right)$,

$$
\beta-\frac{G_{1}-G_{2}}{G_{2}\left(1+\kappa_{1}\right)}, \gamma-\frac{G_{1} \kappa_{2}+G_{2}}{G_{2}\left(1+\kappa_{1}\right)},
$$

$$
\xi-1-\alpha,
$$

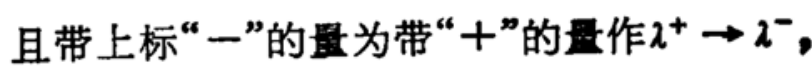
$\boldsymbol{x} \rightarrow-\boldsymbol{\pi}$ 的替换而成.

方程 (3) 给出了奇异指数 $\boldsymbol{\alpha}$ 与材料常数 $\beta 、 \gamma$ 和几何参数 $\lambda^{+} 、 \lambda^{-}$的关系. 通常认为,

\section{- 中国科学院力学研究所LNM资助理逐.}


算指数 $\alpha$ 是方程 (3)的实部最大的根. 实 际上,奇异指数 $\alpha$ 应该有两个. 为揭示这个 特点, 考虑裂纹与相界对称相交即 $\lambda^{+}--\lambda-$ 的情况. 此时, 方程(3)成为 $\left(f^{+}-f^{-}\right) \pm\left(g^{+}\right.$ $\left.-g^{-}\right)=0$. 它对“+”和“一”分别给出一个 $\alpha$. 进一步分析可知, “+”对应着裂纹的张开变
形状态,“一”对应着裂纹的韵切变形状态。因 此，两个奇异指数 $\alpha$ 具有各自的物理含义.

研究表明, 上述结论对于 $\lambda^{+} \neq-\lambda^{-}$的 情况也是成立的.

程品三 段视平 （中国科学院力学研究所，北京 100080)

\section{精确控制 fs 超短光脉冲的同步放大}

研究飞秒脉冲的放大对于发展飞秒超短 脉冲的应用是十分重要的. 在低重复频率下 $(<10 \mathrm{~Hz})$, 用普通的调Q Nd:YAG 激光器 作为 $\mathrm{fs}$ 超短脉冲染料放大器的吾浦源, 会由 于固体器件的不稳定因素, 影响原浦脉冲与 被放大 fs 脉冲之间的同步精度. 同时, 因为 原浦脉冲较宽 ( $10 n s)$, 会使一部分能量浪 费在放大的受激自发辐射 (ASE) 上,从而降 低了信嗓比 ${ }^{(1,2)}$. 因此, 获得窄的原浦脉冲并 控制其与 fs 超短脉冲在染料盒中精确同步, 是设计 $\mathrm{fs}$ 脉冲放大器的关键.

我们首次用腔倒空 Nd:YAG 激光器作 为 $\mathrm{fs}$ 脉冲染料放大器的原浦源, 获得了与 fs 脉冲精确同步的寉原浦脉冲, 并取得了较 好的放大结果. 其方法为: 首先用 $\mathrm{Ar}^{+}$敫 光器票浦的碰撞脉冲锁模环形染料激光器 (CPM Laser) 输出的 fs 脉冲序列产生出一 个相应的电信号序列, 并把这一信号同一台 $\mathrm{Nd}$ : YAG 激光器产生的调 $\mathrm{Q}$ 脉冲相“与”.与 门输出信号再触发 Nd:YAG 激光器的腔倒 空过程, 产生出半宽度为 $5 \mathrm{~ns}$ 的短脉冲. 它 与 $\mathrm{fs}$ 脉冲之间的相对抖动时间小于 $1 \mathrm{~ns}$ 。该 脉冲经放大和倍频后得到波长为 $532 \mathrm{~nm}$ 的 眿冲, 作为 $\mathrm{fs}$ 脉冲染料放大器的泵浦脉冲.
由于辱浦脉冲相应的空间域宽度 ( $5 \mathrm{~ns} \times c$ ) 约 为 $1.5 \mathrm{~m}$, 因此, 可以在电路中用同轴传输线 长度来调节它和 $\mathrm{fs}$ 脉冲在染料盒中的相对 延迟, 而不必采用光学延迟线. 同时, 因为 $\mathrm{CPM}$ 敫光器输出的重复频率为 $85 \mathrm{MHz}, \mathrm{fs}$ 光 脉冲的间隔约为 $12 \mathrm{~ns}$, 染料放大器能够很容 易在 fs 脉冲序列中选出单一脉冲给予放大.

我们用二级长 $1.5 \mathrm{~cm}$ 的染料盒对 $\mathrm{CPM}$ 激光器产生的中心波长为 $617.5 \mathrm{~nm}$ 的 $80 \mathrm{fs}$ 脉 冲进行了放大, 获得了峰值功率为 $2 \times 10^{\prime} \mathrm{W}$ 的单脉冲输出. 所用染料为若丹明 640 的乙 二醇溶腹、二级染料的浓度 分别为 $10^{-5}$ 和 $5 \times 10^{-4} \mathrm{~mol} / \mathrm{L}$. 测得放大倍数为 $6.2 \times 10^{4}$.

致谢: 感谢本永春、钱林兴、顾圣如、陆雨田和 吴瑞昆等同志的大力帮助.

\section{参考文 献}

[1] Migus. A. et al., IEEE J.Q.E., QE-18(1982), 101.

[2] Hnilo, A. A. and Martiniez, O. E., IEEE J. Q.E., QE-23(1987), 593.

沈曋汇 何慧娟张影华 刘玉类 肖纲要 王之江 (中国科学院上海光学棉密机棫研究所激光技术 开放研究实验室,上海 201800) 